\title{
Datação absoluta de depósitos quaternários brasileiros por luminescência
}

\author{
Alethéa Ernandes Martins Sallun ${ }^{1,2}$, Kenitiro Suguio ${ }^{2,3}$, Sônia Hatsue Tatumi ${ }^{4}$, Marcio Yee $^{4,5}$, \\ Janaina Santos ${ }^{6} \&$ Alcina Magnólia Franca Barreto 6
}

\begin{abstract}
Resumo As datações absolutas por luminescência de cristais são utilizadas com sucesso em depósitos quaternários por vários pesquisadores de diferentes países. Porém, no Brasil esses métodos são ainda pouco utilizados e mesmo desacreditados por alguns grupos de pesquisa. Os métodos de datação por luminescência são baseados na teoria da interação das radiações ionizantes com a matéria, no caso com os cristais, e se a idade da amostra a ser datada é calculada à partir da medida das concentrações de defeitos induzidos no material por radiações ionizantes. São aqui apresentados, pela primeira vez, dados de radiação cósmica incidente por espectroscopia- $\gamma$, medidos no Brasil, que são informações importantes usadas na determinação de idades obtidas por esses métodos. Neste trabalho apresenta-se de forma objetiva a metodologia e interpretação de idades obtidas por luminescência com o objetivo de difundir a utilidade dessas análises.
\end{abstract}

Palavras-chave: datações por luminescência, radiação cósmica, depósitos quaternários, Brasil.

\begin{abstract}
Luminescence absolute dating of brazilian quaternary deposits. Absolute datings by luminescence of crystals are successfully used in Quaternary deposits by many researchers in different countries. Nevertheless, these methods are still used few times in Brazil and even are unbelieved by some research groups. The luminescence dating methods are based in theory of interaction of ionizing radiations with the matter, and the age of the sample to be dated is calculated from the concentration of defects induced by ionizing radiations in the matter. For the first time, incident cosmic radiation data measured in Brazil are presented, which are important in age determinations made by these methods. The methodology and interpretation of luminescence data are clearly presented in this paper to help the diffusion of usefulness of these methods.
\end{abstract}

Keywords: luminescence dating, cosmic radiation, quaternary deposits, Brazil.

INTRODUÇÃO Com o avanço tecnológico mais acelerado, iniciado no fim do século XIX, surgiram vários métodos para determinação de idades absolutas. Embora a datação absoluta forneça resultado quantitativo aparentemente simples o pesquisador deve, antes de iniciar qualquer interpretação, procurar analisar de forma criteriosa o significado da idade indicada pelo material utilizado.

Atualmente existem mais de 40 métodos aplicáveis na datação de diversos tipos de materiais associados a eventos do Quaternário e dentre eles tem-se os baseados em danos causados por radiação em materiais geológicos (Tab. 1). Esses danos estão relacionados à mudança de algumas propriedades físicas de sólidos não-condutores (alguns minerais) decorrentes da interação com partículas $\alpha$ e $\beta$, radiação- $\gamma$ e raios cósmicos. Parte desses "danos" ou "defeitos" é reversível, mas outra parte é mais estável e aumenta linearmente com o tempo de exposição à radiação e pode ser empregada na datação de eventos geológicos.

As datações absolutas por luminescência de minerais constituem os únicos métodos geocronológicos de determinação da ocorrência de eventos geológicos, que podem ser relacionados às idades de deposição de sedimentos (Duller 2004), que permitem o estudo de amostras sem restos orgânicos. Estes métodos têm demonstrado grande potencialidade na datação de depósitos quaternários, pois alcançam idades muito mais antigas (até cerca de $10^{6}$ anos) que o método do radiocarbono e, portanto, compreende parte importante do Quaternário. A partir da Termoluminescência (TL, em inglês Thermoluminescence $=$ TL), Luminescência Opticamente Estimulada (LOE, em inglês Optically Stimulated Luminescence $=$ OSL) e Luminescência Estimulada por Raios Infravermelhos (LERI, em inglês Infrared Stimulated Luminescence $=$ IRSL) podem ser estabelecidas cronologias absolutas de depósitos quaternários (continentais e marinhos), que testemunharam importantes eventos geológicos e suas gêneses podem ser desvendadas a partir de outros métodos quantitativos.

Os primeiros trabalhos com datação por luminescência surgiram no século passado pelo método TL, que envolveram cerâmica arqueológica na década de 50

1 - Instituto Geológico, São Paulo, SP, Brasil. E-mail: alethea@igeologico.sp.gov.br

2 - Instituto de Geociências, Universidade de São Paulo, São Paulo, SP, Brasil. E-mail: kenitirosuguio@hotmail.com

3 - Centro de Pós-Graduação, Pesquisa e Extensão (CEPPE), Universidade Guarulhos (UnG), Guarulhos, SP, Brasil

4 - Faculdade de Tecnologia de São Paulo, São Paulo, SP, Brasil. E-mail: tatumi@fatecsp.br

5 - Dep. Engenharia Metalúrgica e de Materiais, Escola Politécnica, Universidade de São Paulo, São Paulo, SP, Brasil. marcioye@usp.br

6 - Departamento de Geologia, Universidade Federal de Pernambuco, Recife, PE, Brasil. janainasc@gmail.com, alcina@ufpe.br 
Tabela 1 - Métodos utilizados para datação de material quaternário baseados em danos causados por radiação (modificado de Geyh \& Schleicher 1990).

\begin{tabular}{l|l}
\hline \multicolumn{1}{c|}{ Métodos } & \multicolumn{1}{c}{ Materiais utilizáveis } \\
\hline Termoluminescência (TL) & $\begin{array}{l}\text { Cerâmica arqueológica, cinza de fogueira, material lítico queimado, fósseis, } \\
\text { vidro, carbonato, falha geológica, areia quartzosa e lava vulcânica }\end{array}$ \\
\hline Traço de fissão (TF) & $\begin{array}{l}\text { Minerais (apatita, biotita, epídoto, esfeno, muscovita e zircão), vidro } \\
\text { vulcânico e madeira silicificada }\end{array}$ \\
\hline $\begin{array}{l}\text { Ressonância Paramagnética } \\
\text { Eletrônica (RPE) }\end{array}$ & $\begin{array}{l}\text { Espeleotema (carbonato secundário), quartzo, sílex, gipsita, conchas de } \\
\text { moluscos marinhos e ossos }\end{array}$ \\
\hline $\begin{array}{l}\text { Traço de Recolhimento de } \\
\text { Partícula- } \alpha\end{array}$ & Minerais (mica) e ossos \\
\hline Exo-elétrons (EETE) & Ossos \\
\hline $\begin{array}{l}\text { Corrente Termicamente Estimulada } \\
\text { (CTE) }\end{array}$ & Basalto \\
\hline $\begin{array}{l}\text { Luminescência Estimulada por } \\
\text { Raios Infravermelhos (LERI) }\end{array}$ & Feldspato \\
\hline $\begin{array}{l}\text { Luminescência Opticamente } \\
\text { Estimulada (LOE) }\end{array}$ & Partículas minerais sedimentares expostas à luz solar por poucos minutos \\
\hline
\end{tabular}

(Grögler et al. 1958), e por LOE na década de 80 (Huntley 1985). A aplicação na determinação de idade de depósitos sedimentares iniciou-se mais intensamente na década de 80 (Wintle \& Huntley 1980).

Numerosos artigos descrevem detalhadamente as metodologias para preparação e datação de diferentes tipos de cristais (Wintle 1997). Com os avanços tecnológicos das últimas décadas, novos métodos e descobertas aumentaram a confiabilidade das idades obtidas por luminescência (Duller 2004). Os minerais usados na datação são comumente quartzo e feldspato, e potencialmente zircão, vidro vulcânico, carbonato e fluorapatita (Bailiff 1976, Ugumori \& Ikeya 1980, Berger \& Huntley 1983, Aitken 1985 e 1998, Wintle 1997) e olivina (Takada et al. 2006).

MÉTODO DA LUMINESCÊNCIA A luminescência é uma propriedade física de materiais cristalinos ou vítreos, previamente submetidos a radiações ionizantes (raios cósmicos e isótopos radioativos), de emitir luz em resposta a algum estímulo externo, como por aquecimento até determinadas temperaturas (Termoluminescência - TL), por estímulo óptico (Luminescência Opticamente Estimulada - LOE ou Luminescência Estimulada por Raios Infravermelhos - LERI), por pressão (Triboluminescência), por reações químicas (Quimioluminescência), por radiação eletromagnética (Radioluminescência) ou por radiação ionizante (Fotoluminescência) (Lian \& Roberts 2006).

Os métodos de datação por TL, LOE e LERI são baseados na interação das radiações com a matéria, e fundamentam-se no fato de que as idades dos materiais a serem datados podem ser calculadas pelas medidas das concentrações de defeitos induzidos nos materiais por radiações ionizantes do ambiente deposicional
(Shelkoplyas \& Morozov 1965, Prescott \& Robertson 1997).

Materiais geológicos e arqueológicos compostos de minerais (calcita, fluorita e quartzo) e fragmentos de cerâmica, respectivamente, recebem radiações ionizantes (partículas $\alpha$ e $\beta$ e radiação $\gamma$ ) provenientes de raios cósmicos e da desintegração de isótopos radioativos naturais como $\mathrm{U}^{235}, \mathrm{U}^{238}, \mathrm{Th}^{232} \mathrm{e} \mathrm{K}^{40}$ e seus filhos radioativos, que se encontram no seu interior ou nas vizinhanças. A radiação cósmica influi na intensidade da luminescência que, em geral, é constante perto do Equador, mas como as partículas componentes dos raios cósmicos são atraídas pelo campo geomagnético da Terra, a intensidade torna-se maior nos pólos.

Parte desta radiação provoca ionização por efeito fotoelétrico, efeito Compton e por produção de pares. Quando um cristal iônico sofre ação de radiações ionizantes, pequena parte da energia é usada para produzir defeitos pontuais ou vários tipos de centros, que resultam em aprisionamento de elétrons e lacunas (cargas positivas) em defeitos localizados na banda proibida de energia (BP - em inglês bandgap - BG) no interior de sua rede cristalina (Fig. 1), e originam estados metaestáveis criados por defeitos e impurezas.

Quando o mineral é aquecido termicamente ou estimulado opticamente, os elétrons aprisionados são liberados e podem recombinar-se com defeitos da rede cristalina, emitindo luminescência. O número desses defeitos da rede cristalina, dentro de certos limites, cresce proporcionalmente à dose de radiação incidente e ao tempo de irradiação. Assim, quando se mede a luz emitida por esses centros é possível, por exemplo, determinar quando um mineral se cristalizou ou quando um sedimento foi exposto à luz solar pela última vez durante período mínimo de 6-8 horas (TL) e alguns 


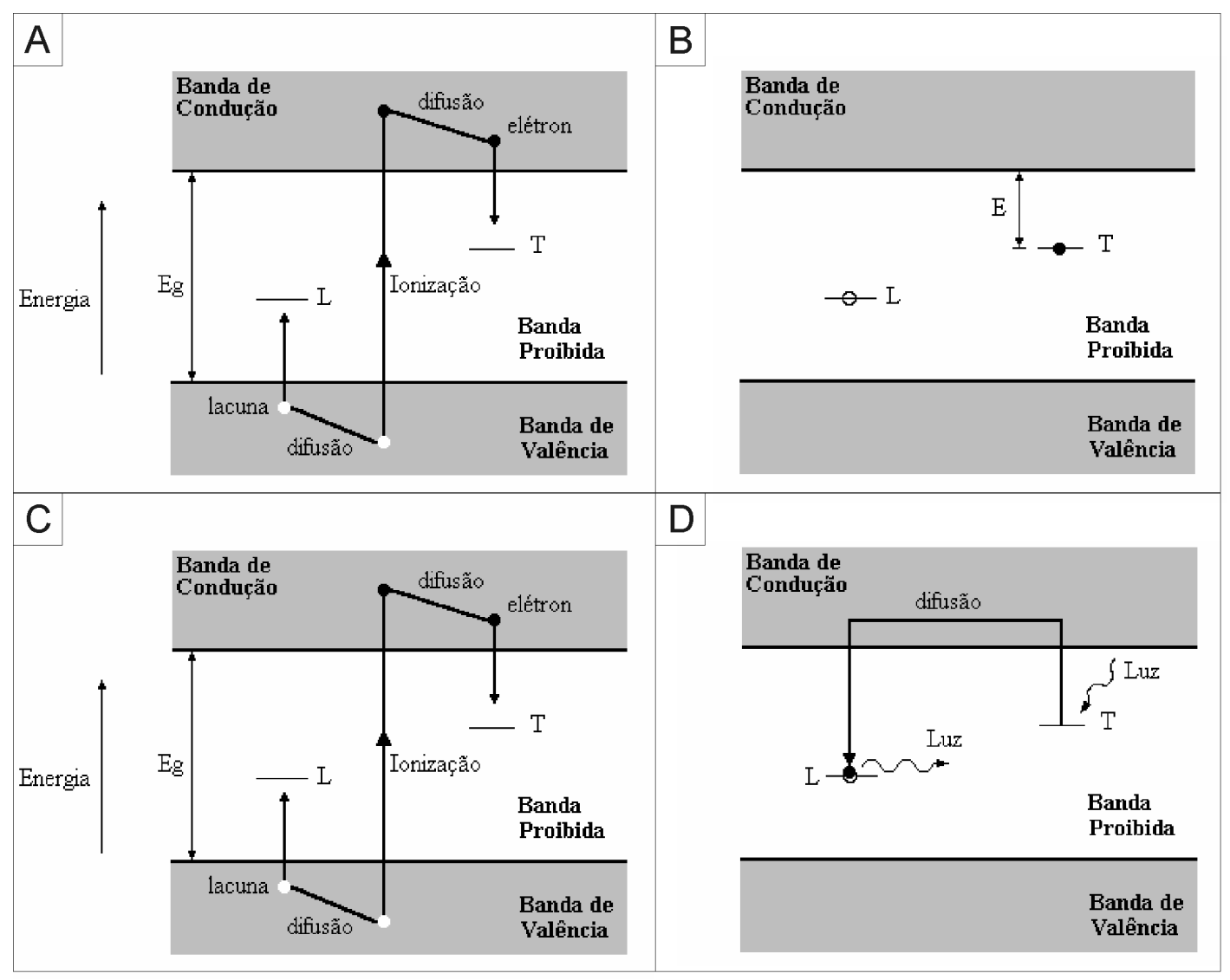

Figura 1 - Modelo de bandas aplicado a um cristal: (A) processo de ionização do cristal, com a liberação de cargas (elétrons e lacunas) da Banda de Valência (BV) para a Banda Proibida (BP); (B) formação dos niveis metaestáveis na BP (nivel Trepresenta elétron aprisionado e nível L representa uma lacuna aprisionada); (C) aquecimento do cristal que provoca liberação de elétron e posterior recombinação do elétron com uma lacuna em L com a liberação de luz TL; (D) estimulação do cristal com luz que provoca a liberação do elétron e posterior recombinação do elétron com uma lacuna em L com a liberação de luz LOE.

minutos (LOE e LERI) segundo Aitken (1985), ou sofreram aquecimento superior a $100{ }^{\circ} \mathrm{C}$ (Aitken 1998). Os métodos de datação por TL, LOE e LERI permitem obter idades desde poucas dezenas de anos até cerca de 1,5 Ma anos, conforme as características das amostras e das sensibilidades dos equipamentos de medida de luminescência. Em geral, idades maiores que 800.000 anos são obtidas somente em grãos de quartzo colocados em ambientes com baixos níveis de radiação ambiental (Huntley et al. 1993a,b).

As idades das amostras são calculadas segundo a equação (1), onde: $\mathrm{I}=$ idade $(\operatorname{anos}) ; \mathrm{P}=$ paleodose (Gy), que corresponde à energia total absorvida pelo cristal pela incidência de radiações ionizantes; $\mathrm{DA}_{\gamma}, \mathrm{DA}_{\beta}$ e $\mathrm{DA}_{\text {r.c. }}=$ doses anuais (Gy/ano) relativas às radiações- $\gamma$, partículas- $\beta$ e aos raios cósmicos, respectivamente.

$$
I=\frac{P}{D A_{\gamma}+D A_{\beta}+D A_{r . c}}
$$

Paleodose (P) Existem várias formas para se deter- minar os valores de paleodoses, mas o método mais utilizado é o da regeneração total. Por este método, a amostra é inicialmente submetida a tratamentos químicos, que variam de acordo com o mineral a ser analisado. São utilizados ácidos fluorídrico (HF) e clorídrico ( $\mathrm{HCl})$ para remoção de matéria orgânica, óxidos e hidróxidos de ferro e carbonatos (Mejdahl 1979) e politungstato de sódio (SPT em inglês) para separar apenas os grãos de quartzo dos minerais pesados e feldspatos. A seguir a amostra é separada em aproximadamente seis partes e uma é submetida à leitura de luminescência para obtenção da luminescência natural, e as outras partes recebem tratamento térmico a $480^{\circ} \mathrm{C}$ por $15 \mathrm{mi}-$ nutos, para eliminar toda a TL ou são expostas à luz solar por cerca de 16 horas para LOE. Em seguida, as amostras são irradiadas para receber doses conhecidas de radiações ionizantes e finalmente são medidas as razões TL/LOE (Fig. 2A). Medidas adicionais podem ser realizadas com LERI para identificar contaminação com feldspato em análises de quartzo.

Com esses resultados é obtida uma curva de calibração para cada amostra, plotando as intensidades de luminescência em função da dose e fixando-se uma 

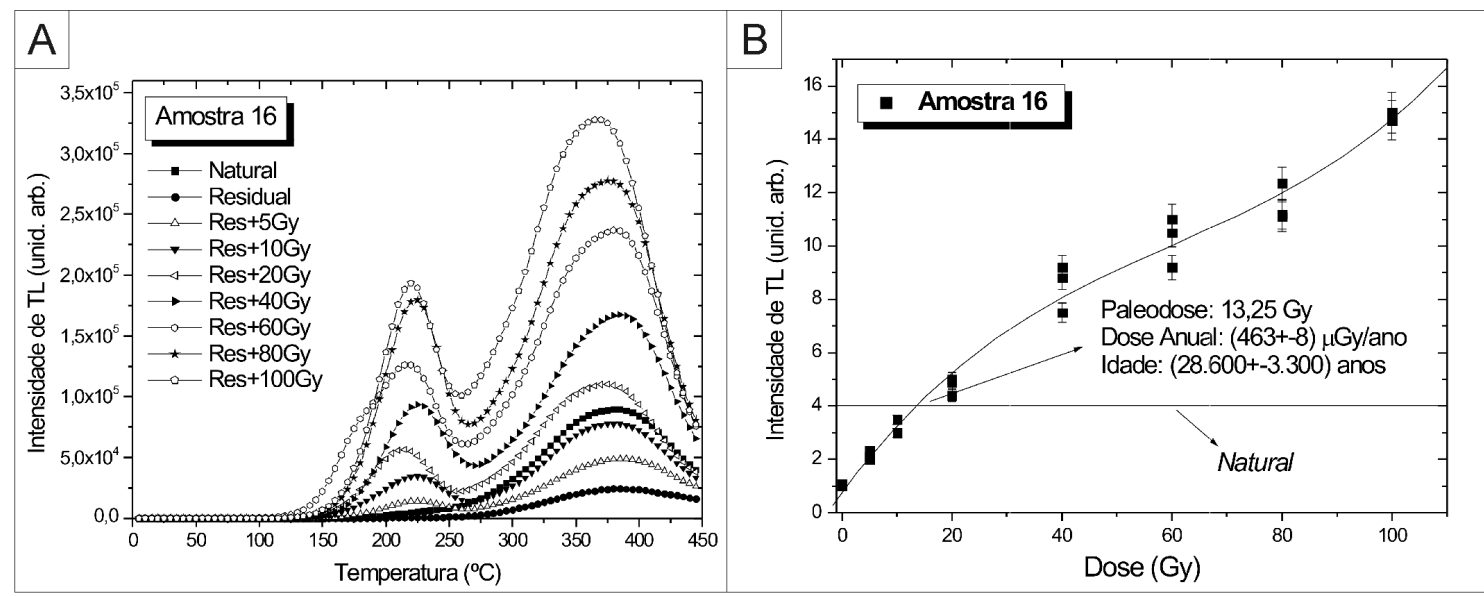

Figura 2 - Exemplos dos dados obtidos para colúvio da Bacia Hidrográfica do Alto Rio Paraná: (A) Curvas de emissão de TL de grãos de quartzo em amostra natural submetida a doses diferentes; (B) Curva de calibração de grãos de quartzo pelo método da regeneração total, à temperatura em torno de $375^{\circ} \mathrm{C}$.

temperatura (Fig. 2B). Para o cálculo da idade é obtido $\mathrm{o}$ valor de $\mathrm{P}$, pela intersecção da curva de calibração com a linha que representa a luminescência natural, e das doses anuais (Das), através da determinação dos teores de $\mathrm{U}^{235} / \mathrm{U}^{238}, \mathrm{Th}^{232}, \mathrm{~K}^{40}$ por espectroscopia- $\gamma$ da própria amostra, utilizando-se detectores de germânio ou de $\mathrm{NaI}(\mathrm{Tl})$.

RAIOS CÓSMICOS Os raios cósmicos primários originam-se fora do Sistema Solar e são predominantemente compostos por prótons com mistura de núcleos com grande massa atômica. A energia média é de alguns $\mathrm{GeV}$ e a intensidade do espectro decai rapidamente à medida que a energia aumenta. $\mathrm{O}$ campo geomagnético é suficiente para interferir nas partículas de baixa energia e, desta forma, a intensidade de raios cósmicos que atingem o topo da atmosfera é maior perto dos pólos. O fluxo de raios cósmicos depende do campo geomagnético e também da atmosfera.

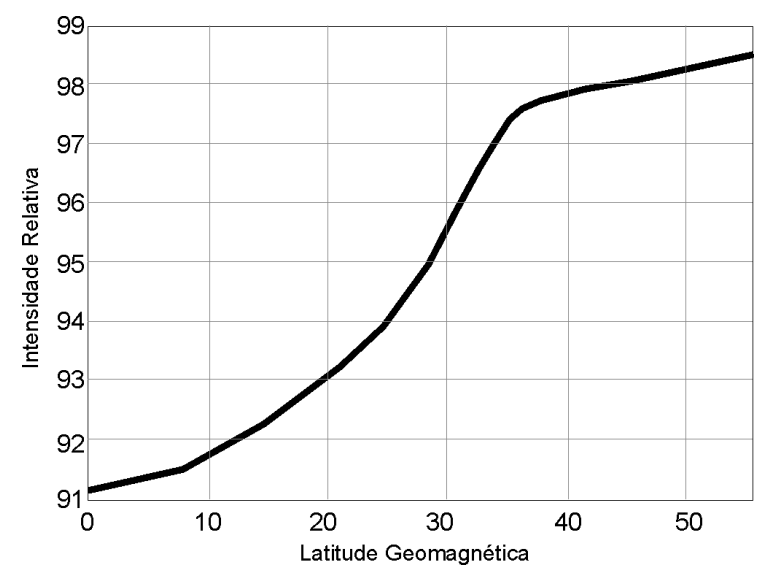

Figura 3 - Efeito da latitude geomagnética, entre 0 a $55^{\circ}$, nas intensidades relativas de raios cósmicos em função da latitude (Prescott \& Stephan 1982).
Partículas com energias menores que $2,2 \mathrm{GeV}$ não conseguem penetrar e atingir o nível do mar, devido à densidade da atmosfera. Isto significa que quando se move para os pólos, saindo do Equador, a radiação cósmica aumenta com a latitude até chegar a um valor constante nos pólos. Em relação à longitude a variação é pequena (em torno de 7\%), pois o momento magnético não coincide com o eixo do spin da Terra. As intensidades relativas dos raios cósmicos (Fig. 3) aumentam de aproximadamente $6,5 \%$ até $40^{\circ}$, permanecendo praticamente constante para latitudes maiores (Prescott \& Stephan 1982).

Após penetrar na atmosfera os raios cósmicos interagem com as moléculas do ar atmosférico, provocam ionizações em cadeia e produzem prótons, múons $(\mu)$, nêutrons e outras partículas nucleares. Os raios cósmicos que chegam à superfície da Terra são divididos em duas componentes: as radiações de baixa energia compostas, na sua maioria, por elétrons que são absorvidos após penetrarem cerca de $0,5 \mathrm{~m}$ no solo, e as radiações de alta energia que consistem predominantemente de múons.

Os depósitos sedimentares recebem radiações ionizantes a partir de raios cósmicos e da desintegração de isótopos radioativos naturais. A exatidão desses valores é de extrema importância para se obter valores mais confiáveis, já que as idades de amostras obtidas por luminescência são inversamente proporcionais à dose da radiação cósmica incidente no local.

Valor Teórico da Radiação Cósmica As intensidades das radiações cósmicas podem ser calculadas teoricamente a partir da altitude, latitude e tipo de rocha. A idade da amostra é calculada segundo a equação abaixo (2), onde: $\mathrm{K}=$ a partir da densidade da rocha (mrad/ ano); F, J e H são constantes que dependem do valor da latitude geomagnética do local de medida e podem ser determinadas através da figura $4, \mathrm{e} h=$ altitude. 


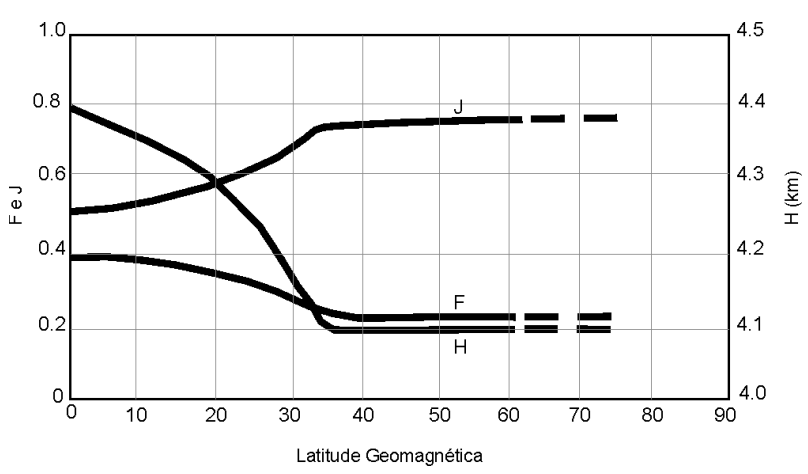

Figura 4-Os fatores F, Je H dependem das latitudes geomagnéticas (Prescott \& Stephan 1982).

$$
I=K\left[F+J \exp \left(\frac{h}{H}\right)\right]
$$

Medida Experimental da Radiação Cósmica A primeira dosimetria in situ de radiação cósmica por espectrometria- $\gamma$, para datação de sedimentos no Brasil, foi realizada no Rio Paraná, no reservatório da Usina Hidrelétrica Engenheiro Sérgio Motta (UHESM - Porto Primavera) em Presidente Epitácio (SP), em fevereiro de 2005 (Sallun et al. 2006). O local escolhido para as medidas foi a porção relativamente mais profunda do reservatório, com lâmina d'água de 9 - 10 m, para evitar a possível interferência de radiações provenientes de isótopos radioativos do solo $\left(\mathrm{U}^{235}, \mathrm{U}^{238}, \mathrm{Th}^{232}\right.$ e $\left.\mathrm{K}^{40}\right)$ nas medidas. Foram obtidos dados em três pontos dis- tintos (Tab. 2), sendo dois pontos em barco sobre o Rio Paraná (Fig. 5A) e um ponto para controle, em terraço da margem esquerda do Rio Paraná.

Recentemente foi realizada uma segunda dosimetria in situ de radiação cósmica por espectrometria- $\gamma$ no reservatório Petrônio Portela, açude construído no leito do Rio Piauí, em São Raimundo Nonato (PI) em julho de 2006. O local escolhido para as medidas foi a porção relativamente mais profunda do reservatório, com lâmina d'água de aproximadamente $20 \mathrm{~m}$. Foram obtidos dados em quatro pontos distintos (Tab. 2), sendo dois pontos em barco sobre o Rio Piauí (Fig. 5B) e dois pontos para controle, em dois sítios arqueológicos da Serra da Capivara (abrigo arenítico da Toca do Sítio do Meio e a caverna calcária da Toca de Cima dos Pilão).

As radiações cósmicas médias experimentais foram de 174,22 $\mu \mathrm{Gy} /$ ano e $200,18 \mu \mathrm{Gy} / \mathrm{ano}$, enquanto que as radiações cósmicas médias teóricas, calculadas pela equação 2, foram de 209,85 $\mu \mathrm{Gy} /$ ano e 250,00 $\mu \mathrm{Gy} /$ ano nas regiões dos rios Paraná e Piauí, respectivamente.

Os valores obtidos nos sítios arqueológicos são muito superiores aos obtidos no Rio Piaú, devido à contribuição maior da rocha das paredes das cavidades. A diferença obtida entre as medidas realizadas nas cavidades provavelmente está relacionada aos tipos distintos de composição da rocha formadora, arenítica e calcária.

A diferença entre a média experimental e teórica de cerca de $20 \%$ para as regiões do Rio Paraná e Rio Piauí introduziriam erros proporcionais nas idades obtidas por luminescência. Esse fato mostra a neces-

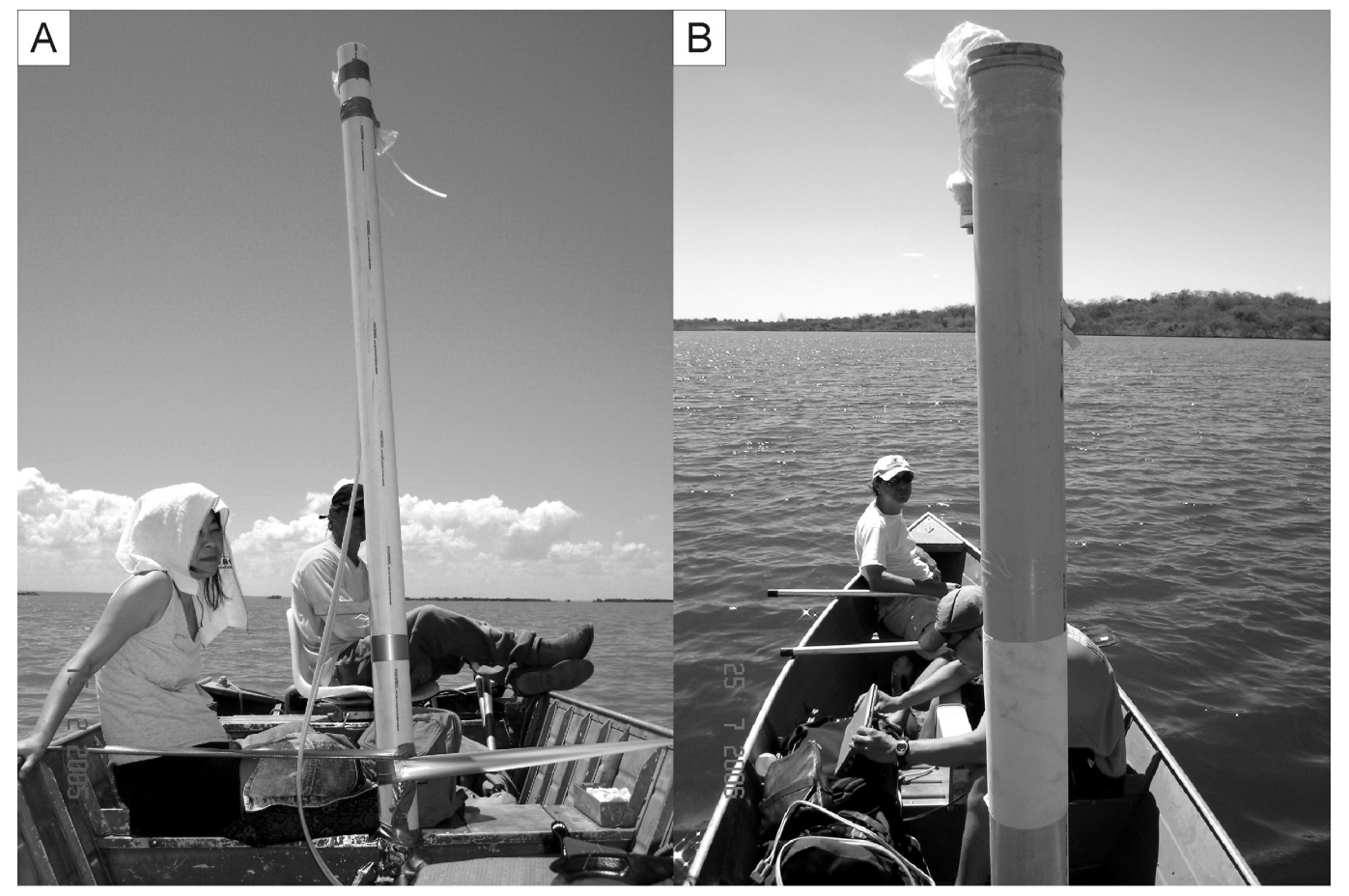

Figura 5 - Medições de radiação cósmica realizadas em barco no Rio Paraná (A) e no Rio Piauí (B). 
Tabela 2 - Coordenadas geográficas dos locais de medida, tempos de medida e doses obtidas $\left(D A_{r . c .}=\right.$ dose anual proveniente de raios cósmicos, $D A_{\text {solo }}=$ dose anual proveniente do solo, * medidas efetuadas no solo, ${ }^{1}$ Toca do Sítio do Meio e ${ }^{2}$ Toca de Cima dos Pilão), com valores médios experimentais e teóricos das radiações cósmicas para cada local.

\begin{tabular}{|c|c|c|c|c|}
\hline Coordenadas geográficas & $\begin{array}{l}\text { Início de medida } \\
\text { (horas) }\end{array}$ & $\begin{array}{c}\text { Tempo de contagem } \\
\text { (segundos) }\end{array}$ & $\begin{array}{c}\mathrm{DA}_{\text {r.c. }} \\
(\mu \mathrm{Gy} / \mathrm{ano})\end{array}$ & $\begin{array}{c}\mathrm{DA}_{\text {solo }} \\
\mu \mathrm{Gy} / \mathrm{ano})\end{array}$ \\
\hline \multicolumn{5}{|c|}{ Rio Paraná (SP) } \\
\hline $21^{\circ} 44^{\prime} 31^{\prime \prime} \mathrm{S} / 52^{\circ} 06^{\prime} 58,6^{\prime \prime} \mathrm{W}$ & 08:05 & $5.400,79$ & 175,29 & - \\
\hline $21^{\circ} 45^{\prime} 10^{\prime} \mathrm{S} / 52^{\circ} 05^{\prime} 49,5^{\prime \prime} \mathrm{W}$ & $11: 30$ & $5.402,16$ & $221,61 *$ & $46,32 *$ \\
\hline $21^{\circ} 44^{\prime} 53^{\prime \prime} \mathrm{S} / 52^{\circ} 06^{\prime} 30,1^{\prime \prime} \mathrm{W}$ & $14: 16$ & $5.400,74$ & 173,15 & - \\
\hline \multicolumn{3}{|c|}{ Valor médio experimental } & 174,22 & - \\
\hline \multicolumn{3}{|c|}{ Valor médio teórico } & 209,85 & - \\
\hline \multicolumn{5}{|c|}{ Rio Piauí (PI) } \\
\hline $09^{\circ} 00^{\prime} 6,8^{\prime \prime} \mathrm{S} / 42^{\circ} 26^{\prime} 11,9^{\prime \prime} \mathrm{W}$ & $11: 33$ & $14.662,64$ & 210,44 & - \\
\hline $09^{\circ} 00^{\prime} 6,8^{\prime \prime} \mathrm{S} / 42^{\circ} 26^{\prime} 11,9^{\prime \prime} \mathrm{W}$ & $9: 20$ & $9.939,84$ & 189,92 & - \\
\hline $08^{\circ} 49^{\prime} 44,6 ” \mathrm{~S} / 42^{\circ} 32^{\prime} 40,1$ '” & $14: 29$ & $3.602,88$ & - & $2.180,80 * l$ \\
\hline $08^{\circ} 51^{\prime} 50,9^{\prime \prime} \mathrm{S} / 42^{\circ} 33^{\prime} 23,9^{\prime \prime} \mathrm{W}$ & $16: 20$ & $3.604,46$ & - & $3.077,90 * 2$ \\
\hline \multicolumn{3}{|c|}{ Valor médio experimental } & 200,18 & - \\
\hline \multicolumn{3}{|c|}{ Valor médio teórico } & 250,00 & - \\
\hline
\end{tabular}

sidade de novas medidas de radiação cósmica, em outras regiões do Brasil, para que as idades determinadas por luminescência sejam mais precisas e confiáveis. As causas destas diferenças estão também ligadas a variáveis, tais como, época do ano, clima, hora e dia, que não são consideradas no cálculo teórico, mas interferem na medida experimental.

CONFIABILIDADE NA DATAÇÃO POR LUMINESCÊNCIA Os significados das idades obtidas por luminescência devem ser analisados com cuidado pelos pesquisadores. As composições química e mineralógica e a origem dos sedimentos podem interferir na confiabilidade dos resultados obtidos.

Coleta de amostras Antes da coleta de amostras o estado de preservação do sedimento deve ser investigado, de modo que a idade obtida esteja ligada à deposição. Depois, devem ser definidos os níveis de coleta de amostras e escavada uma trincheira rasa (alguns centímetros de profundidade) para remoção da parte superficial, que poderia estar contaminado com material caído de cima. Recomenda-se a coleta em um local do depósito sedimentar onde a litologia é homogênea em um raio de pelo menos $30 \mathrm{~cm}$ distante do local de coleta. O nível mais superficial de coleta deve situar-se, no mínimo, 0,5 a $1 \mathrm{~m}$ de profundidade para tentar evitar materiais de exposição recente aos raios solares, bem como horizontes bioturbados por animais (zoorturbações) e plantas (fitoturbações), e horizontes pedogenéticos. As amostras devem ser coletadas em tubos opacos (geralmente de PVC ou de alumínio), de cerca de $30 \mathrm{~cm}$ de comprimento por $5 \mathrm{~cm}$ de diâmetro.

Os tubos devem ser introduzidos horizontalmente em níveis de coleta por percussão com marreta ou em subsuperfície ser utilizada sondagem mecanizada, que permita recuperação de testemunhos. Logo após a retirada, as extremidades dos tubos devem ser fechadas para evitar a exposição à luz solar e enviados imediatamente ao laboratório, antes da perda de umidade.

As coletas em sacos plásticos pretos podem facilitar a contaminação, que aumentará o erro nas idades dos sedimentos, por exposição à radiação solar ou pela perda de umidade. No laboratório os tubos são abertos e desprezados os sedimentos das duas extremidades, que podem ter sido expostas aos raios solares.

Exposição à radiação solar As idades de sedimentos quaternários, obtidas por luminescência, representam o tempo decorrido após a última exposição dos grãos à luz solar, desde que ela tenha sido suficiente para eliminação da luminescência adquirida previamente. Porem, é difícil saber se as idades correspondem a luminescência posterior à última deposição, exceto em amostras de depósitos com idades conhecidas por outros métodos de datação absoluta. São quatro os processos que podem "zerar" as idades de grãos de quartzo, pela eliminação total do sinal prévio de luminescência: formação de novo mineral por cristalização a partir do magma ou por processos bioquímicos, submissão do cristal à pressão, aquecimento de cristal até 200 a $500{ }^{\circ} \mathrm{C}$ ou 
exposição do cristal à luz solar (Duller 2004).

Em geral, a intensidade do sinal de luminescência aumenta proporcionalmente ao aumento da intensidade de radiação. Entretanto, as relações entre as doses de exposição e as intensidades de luminescência não são lineares, pois os sinais crescem assintoticamente até um nível máximo e, portanto, cada amostra requer calibração independente.

A presença de minerais, nas formas de inclusões ou nas superfícies de grãos analisados por luminescência, pode afetar a idade obtida (De Corte et al. 2006). Grãos minerais naturais podem exibir películas superficiais de óxidos e hidróxidos de ferro ou manganês e argilominerais, que podem obliterar parcialmente a passagem de luz solar (Murray \& Olley 2002). A presença de minerais acessórios (óxidos de ferro, titânio e zircônio), bem como de inclusões em grãos de quartzo, principalmente com concentração de U e Th, pode fornecer idades superestimadas.

Mesmo durante o transporte eólico de grãos minerais ocorre atenuação de exposição à luz solar, mas os carreados por transporte subaquoso são mais problemáticos (Wintle \& Huntley 1982, Berger \& Busacca 1995, Olley et al. 1996), pois o efeito da luz incidente é praticamente neutralizado no primeiro metro de lâmina d'água (Oliver 1990).

$\mathrm{O}$ intenso intemperismo químico, que promove a dissolução de minerais primários e a formação de minerais secundários de granulação fina, seguido de sua migração vertical e acumulação em níveis inferiores, pode afetar os valores das doses de radiação (Aitken 1998, Wagner 1998). Durante a evolução de um perfil de alteração por intemperismo, o surgimento de poros e o seu preenchimento por argilominerais pode causar mudanças na concentração de elementos radioativos (Jeong et al. 2007). O intemperismo é também capaz de produzir defeitos em cristais de quartzo e a idade determinada poderia corresponder a do último intemperismo intenso, sem posterior exposição à luz solar (Aitken 1985). Porém, os efeitos de exposição à radiação solar sobrepujam os do intemperismo e, portanto, as curvas de crescimento correspondem principalmente à intensidade de luz solar recebida.

Estudos de Porat et al. (2007) indicam que o mecanismo de fluidificação de sedimentos, com conseqüente injeção de diques clásticos durante atividades sísmicas, pode "zerar" o sinal de luminescência de minerais. Em região submetida à atividade tectônica, com eventual reativação de falhas, pode ocorrer aumento de temperatura que poderá eliminar a luminescência previamente adquirida.

Em minerais com inclusões fluídas, os aumentos de temperaturas podem interferir nos resultados de TL, pois podem ocorrer trocas de elementos-traço entre as fases líquida e sólida, que interfeririam nos resultados das análises (Miallier et al. 2001).

Obtenção da Luminescência As leituras de luminescência de amostras podem ser feitas por dois métodos distintos: de alíquota múltipla (em inglês multiple ali- quot regenerative-dose $=$ MAR) e de alíquota única (em inglês single aliquot regenerative-dose $=\mathrm{SAR}$ ), segundo Murray \& Wintle (2000) e Wintle \& Murray (2006). O mais utilizado é o método MAR, onde as medições são feitas em cerca de 20 a 40 grãos minerais, nos quais são aplicadas diferentes doses de radiação em laboratório e os resultados permitem delinear uma "curva de crescimento", que representa a resposta da luminescência do material à radiação (Li \& Wintle 1992). Porém, esses grãos podem ter sido expostos heterogeneamente à luz solar e, portanto, alguns grãos podem ter sido "zerados" e outros não. Não há um consenso sobre como deve ser feita a normalização das medidas obtidas em grãos e a idade aparente representaria o valor médio de luminescência de todos os grãos. Como grãos "nãozerados" estariam misturados com "zerados" a média sempre tende a ser maior, que a idade de última exposição solar. Pelo método SAR muitas medidas de luminescência são feitas sobre grãos individuais de única amostra, que permitiria diminuir o erro nas medidas e identificar se o sinal de luminescência foi completamente "zerado" em todos os grãos minerais.

Devido ao maior tempo necessário nas medidas pelo método SAR que, portanto, é mais caro o método MAR é o mais utilizado, principalmente quando se quer fazer mais de uma estimativa para cada amostra. Os ruídos introduzidos nas medidas podem ser identificados e analisados a partir da "curva de crescimento", após executar duas a três medidas para cada dose. Por exemplo, quando os dados obtidos pelo método MAR estão muito dispersos, devem ser realizadas medidas pelo método SAR para aumentar a confibialidade nas idades medidas. Algumas análises pelo método SAR também podem ser feitas em conjuntos de amostras submetidos ao método MAR, mesmo que as "curvas de crescimento" sejam de boa qualidade, de modo a aumentar confiabilidade nos dados obtidos.

Importantes informações paleoambientais têm sido obtidas pelo uso simultâneo dos métodos MAR e SAR nas mesmas amostras. A dispersão dos sinais de luminescência indica que o sedimento não foi comple-

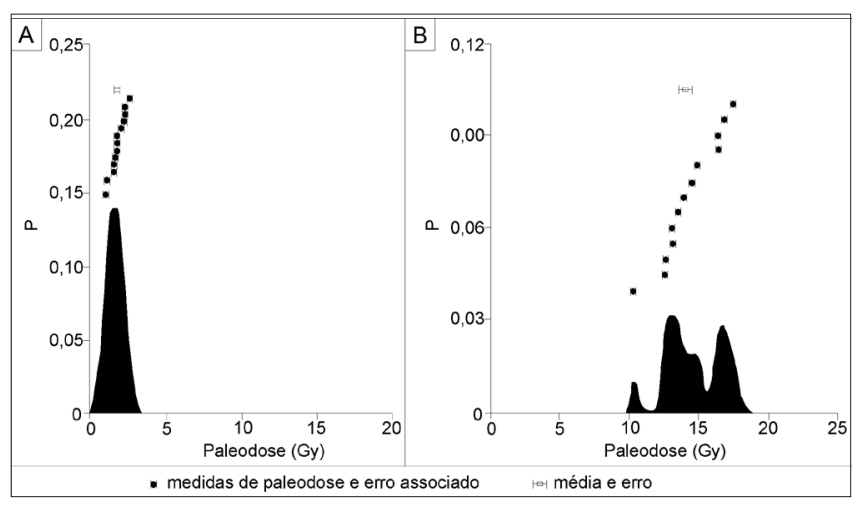

Figura 6 - Exemplos de medidas por alíquotas múltiplas (MAR) de LOE apresentados por Bateman et al (2007) de amostra de dunas típicas fósseis (A) e lineares de Tsodilo Hills (B), em Botswana (Thomas et al. 2003). 
tamente exposto à luz solar, isto é, teria ocorrido deposição rápida (Srivastava et al. 2006) ou a concentração de finos em suspensão na lâmina d'água teria sido alta, causando aumento de turbidez (Gemmell 1994, Rhodes \& Pownall 1994).

Mistura de grãos Após a sedimentação os depósitos quaternários freqüentemente podem ser mais ou menos facilmente retrabalhados, pois geralmente são inconsolidados. As bioturbações, tanto fitoturbações como zooturbações, podem causar exumação, bem como revolvimento e mistura de materiais de diferentes profundidades (Hole 1981). O uso de várias técnicas de medidas de luminescência constitui uma estratégia para reconhecer a possível existência de bioturbação em sedimentos, quando faltam evidências físicas detectáveis em campo. Se for encontrada luminescência anômala em determinada amostra, poderia ser sugerida a perturbação pós-deposicional dos sedimentos (Fig. 6). A bioturbação pode expor grãos minerais na superfície e "zerar" a luminescência e, portanto, causar inversão de idade nos depósitos (Li 1994, Bateman et al. 2003, Bateman et al. 2007).

As idades obtidas por luminescência, em grãos de horizontes bioturbados, podem determinar o momento de formação de paleo-horizontes pedológicos, que atualmente podem estar soterrados (Goble et al. 2004).

Decaimento anômalo No método da TL, como a idade é baseada em alguns grãos minerais, ela pode ser subestimada segundo Wallinga et al. (2001), pela existência do fenômeno denominado de "decaimento anômalo" (em inglês = anomalous fading). Este fenômeno é devido ao rápido decaimento nos picos dos sinais de luminescência em temperatura ambiente, contrariamente à estabilidade admitida pelos modelos cinéticos para TL (Tsirliganis et al. 2007). Este fato é verificado principalmente em feldspatos de origem ígnea, mas também foi observado em quartzo vulcânico de mag-

Tabela 3 - Exemplos brasileiros de trabalhos, que empregam a luminescência em materiais geológicos, para datação de depósitos quaternários.

\begin{tabular}{|c|c|c|c|c|c|}
\hline $\begin{array}{l}\text { Tipo de } \\
\text { depósito }\end{array}$ & Área de estudo & $\begin{array}{c}\text { Método } \\
\text { usado }\end{array}$ & $\begin{array}{c}\text { Mineral } \\
\text { usado }\end{array}$ & $\begin{array}{l}\text { Idades obtidas } \\
\mathrm{x} 10^{3} \text { anos A.P. }\end{array}$ & Referência \\
\hline coluvial & Marília e Presidente Prudente (SP) & $\begin{array}{c}\text { TL } \\
\text { LOE }\end{array}$ & quartzo & 935 a 9 & Tatumi et al. (2006) \\
\hline $\begin{array}{l}\text { coluvial } \\
\text { e aluvial }\end{array}$ & Serra da Capivara (PI) & $\begin{array}{c}\mathrm{TL} \\
\mathrm{LOE} \\
\end{array}$ & $\begin{array}{l}\text { quartzo } \\
\text { feldspato }\end{array}$ & 3,3 a 400 & Santos et al. (2005) \\
\hline aluvial & Alto Rio Paraná (SP, MS e PR) & $\begin{array}{c}\mathrm{TL} \\
\mathrm{LOE}\end{array}$ & quartzo & 1,5 a 240 & $\begin{array}{c}\text { Stevaux (1993 e 2000) } \\
\text { Sallun \& Suguio (2006) }\end{array}$ \\
\hline eólico & Rio São Francisco (BA) & $\mathrm{TL}$ & quartzo & 0,9 a 28 & Barreto et al. (2002a) \\
\hline marinho & Juréia (SP) & $\begin{array}{c}\text { TL } \\
\text { LOE }\end{array}$ & $\begin{array}{l}\text { quartzo } \\
\text { feldspato }\end{array}$ & 10,3 a 0,317 & Bentz (2004) \\
\hline marinho & Ilha Comprida (SP) & TL & quartzo & 84,8 a 15,9 & Suguio et al. (2003) \\
\hline marinho & Ilha Comprida (SP) & TL & quartzo & 14 a 62 & Giannini et al. (2003) \\
\hline $\begin{array}{c}\text { eólico } \\
\text { e aluvial }\end{array}$ & Amazônia & $\mathrm{TL}$ & quartzo & 32,6 a 7,8 & Carneiro Filho et al. (2002) \\
\hline marinho & Natal e Macau (RN) & $\begin{array}{c}\text { TL } \\
\text { LOE }\end{array}$ & $\begin{array}{l}\text { quartzo } \\
\text { feldspato }\end{array}$ & 215 a 110 & Barreto et al. (2002b) \\
\hline eólico & $\mathrm{RN}$ & $\begin{array}{c}\text { TL } \\
\text { LOE }\end{array}$ & quartzo & 390 ao presente & Barreto et al. (2004) \\
\hline $\begin{array}{c}\text { coluvial } \\
\text { aluvial }\end{array}$ & SP, MG e RJ & LOE & feldspato & 6,5 a 97,2 & Tatumi et al. (2003a) \\
\hline $\begin{array}{l}\text { eólico } \\
\text { e marinho }\end{array}$ & PB & $\begin{array}{c}\text { TL } \\
\text { LOE }\end{array}$ & quartzo & 3,2 a 229 & Tatumi et al (2003b) \\
\hline $\begin{array}{l}\text { coluvial } \\
\text { e aluvial }\end{array}$ & Queluz (SP) & LOE & feldspato & 33 a 0,65 & Arruda (2004) \\
\hline aluvial & Candelária (RS) & TL & quartzo & 60 & Fett Júnior \& Oliveira (2005) \\
\hline marinho & Paranaguá (PR) & $\mathrm{TL}$ & quartzo & 122 a 5,845 & Barreto et al. (1999) \\
\hline eólico & Cauípe e Pecém (CE) & TL & quartzo & 2,3 a 1 & Maia et al. (1999) \\
\hline coluvial & Itabaiana e Pilar (PB) & $\begin{array}{c}\text { TL } \\
\text { LOE }\end{array}$ & quartzo & 224,5 a 22 & Corrêa et al. 2005 \\
\hline lagunar & Tramandaí (RS) & $\mathrm{TL}$ & quartzo & 12,6 a 7,4 & Dillenburg (1994) \\
\hline $\begin{array}{l}\text { marinho e } \\
\text { eólico }\end{array}$ & Cidreira e Torres (RS) & $\mathrm{TL}$ & quartzo & 237 a 2,567 & Poupeau et al. (1984 e 1988) \\
\hline marinho & Cananéia (SP) & TL & quartzo & 17 a 110,22 & Watanabe et al. (1997) \\
\hline
\end{tabular}




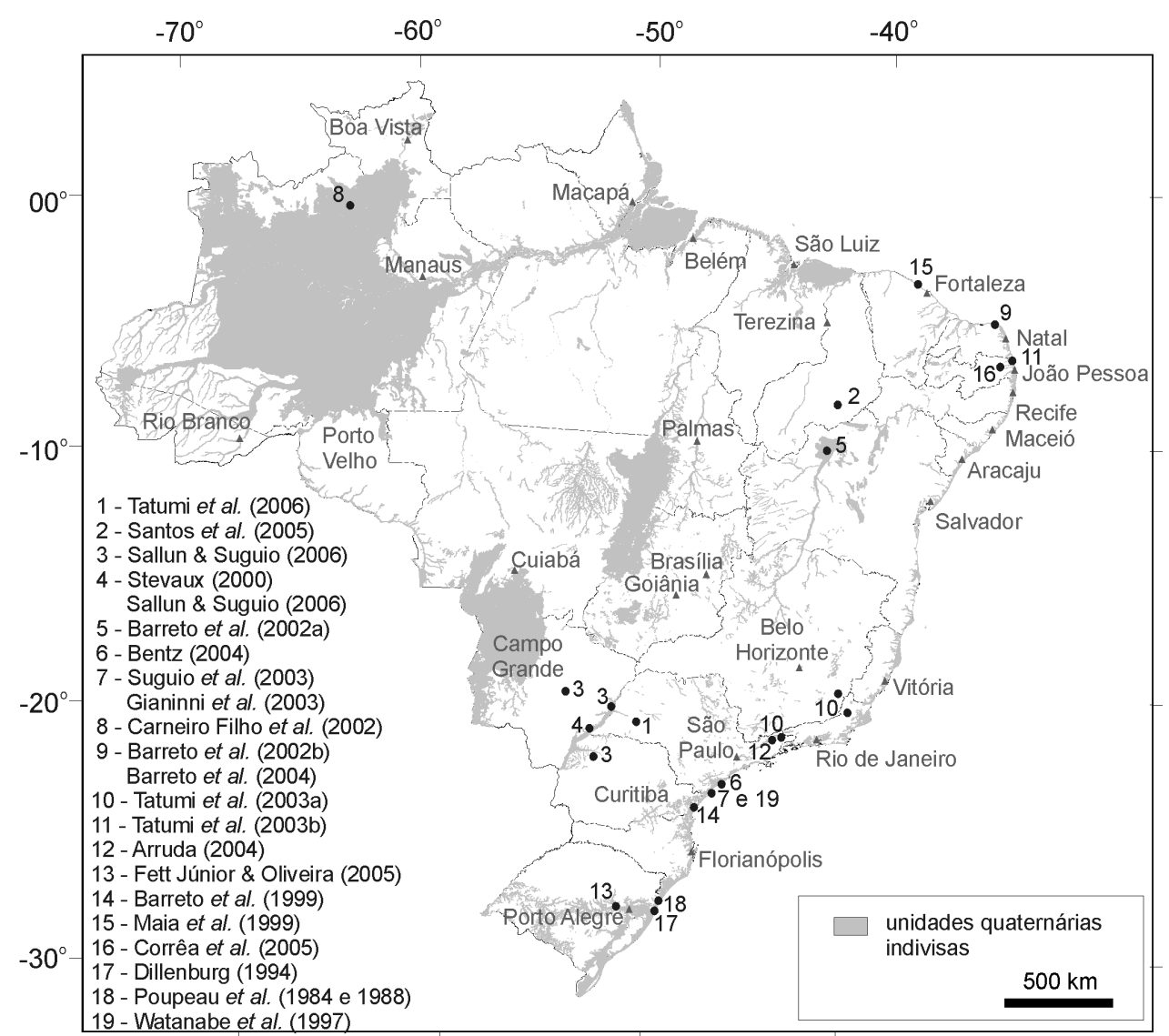

Figura 7 - Localidades no Brasil de datações por luminescência realizadas em sedimentos quaternários no Brasil. Unidades quaternárias obtidas em Bizzi et al. (2001).

mas básicos, em biotita (Li \& Yin 2006), em flogopita (Barcena et al. 1999) e em fluorapatita (Tsirliganis et al. 2007). Este fenômeno ocorre devido ao "Efeito Túnel" da teoria de Mecânica Quântica, onde um elétron tem probabilidade não-nula de sair da armadilha, mesmo sem adquirir muita energia para vencer o potencial.

Existem vários modelos empregados na interpretação deste fenômeno (Chen \& McKeever 1997, Botter-Jensen et al. 2003), porém não ocorre aumento de confiabilidade nas análises. Alguns autores propuseram métodos para determinar identificar a idade correta, em minerais que exibem esse fenômeno, pela medida da taxa de decaimento (Huntley \& Lamothe 2001) e pelo uso de variação da taxa de decaimento medida em diferentes grãos da amostra, para se chegar à idade esperada em grãos sem anomalia (Lamothe \& Auclair 1999 e 2000). Para datação pelo feldspato é utilizada então a LERI (Luminescência Estimulada por Raios Infravermelho), que vem fornecendo resultados mais confiáveis.

DATAÇÕES POR LUMINESCÊNCIA NO BRASIL No Brasil a datação por TL foi aplicada, pela primeira vez, em urnas funerárias indígenas por Szmuk \& Watanabe (1971). Ikeya et al. (1984), Tatumi et al. (1989) e Tatumi (1994) dataram vários espeleotemas provenientes de cavernas do Vale do Ribeira
(SP). Poupeau et al. (1984) e Watanabe et al. (1997), dataram depósitos eólicos e praiais. Dillenburg (1994) datou depósitos lagunares e Barreto (1996) datou areias quartzosas de dunas eólicas inativas do médio Rio São Francisco (BA). No século atual, o emprego deste método de datação intensificou-se no Brasil (Tab. 3 e Fig. 7), porém de forma ainda modesta, quando comparada aos Estados Unidos, Austrália e China.

No Brasil as datações são rotineiramente realizadas somente no LVD (Laboratório de Vidros e Datação) da FATEC-SP (Faculdade de Tecnologia de São Paulo), sob coordenação da Profa. Dra. Sônia Hatsue Tatumi, e no IF (Instituto de Física) da USP (Universidade de São Paulo) realizadas pelo Prof. Dr. Shigueo Watanabe.

No LVD da FATEC-SP é utilizado o aparelho denominado Automated Systems, Model 1100-series Daybreak Nuclear Instruments Inc., com taxa de aquecimento de $10^{\circ} \mathrm{C} / \mathrm{s}$. Segundo o protocolo desenvolvido neste laboratório, os sedimentos quaternários são peneirados para separar grãos com diâmetros entre 88$180 \mu \mathrm{m}$ (areias muito fina a fina), que são submetidos a tratamento químico específico, conforme o tipo de mineral a ser analisado. Para grãos de quartzo são usados ácidos fluorídrico (HF) e clorídrico (HCl) concentrados, para dissolução da porção superficial do grãos e contribuição na redução das doses de $\beta$ e $\alpha$. Cerca de 
40 mg de grãos são enviados para o IPEN-CNEN (Instituto de Pesquisas Energéticas e Nucleares - Comissão Nacional de Energia Nuclear) para irradiação com ${ }^{60} \mathrm{Co}$. A dose acumulada (P) é obtida através do Método de Regeneração Total e os valores das doses anuais (DA) são calculados à partir das concentrações de ${ }^{40} \mathrm{~K},{ }^{232} \mathrm{Th}$, ${ }^{238} \mathrm{U}$ e ${ }^{235} \mathrm{U}$ medidas por espectroscopia- $\gamma$.

CONCLUSÕES As idades obtidas por luminescência de cristais em sedimentos são mundialmente aceitas no meio científico nos dias atuais. Porém, esses métodos ainda são pouco empregados no Brasil pela falta de informações sobre a sua aplicabilidade. Porém, tanto pelo alcance (pelo menos $1 \mathrm{Ma}$ ) como pelo baixo custo, e pela possibilidade de uso de diferentes minerais, constitui uma importante ferramenta na datação do Período Quaternário, principalmente em sedimentos desprovidos de matéria orgânica.

Os depósitos quaternários apresentam gran- de potencial para aplicação do método de datação por luminescência, que conduzirá à melhor reconstituição cronológica dos eventos ocorridos. Adicionalmente, para obter maior precisão nas idades por luminescência, devem ser realizadas novas medidas de radiação cósmica em diversas regiões do Brasil, pois as realizadas em Presidente Epitácio (SP) e em São Raimundo Nonato (PI) apresentam diferenças em relação aos valores teóricos calculados e, deste modo, interferem nas idades das amostras.

Agradecimentos À FAPESP, pela concessão de Bolsa de Doutorado (Processos 03/01737-5) a Alethéa Ernandes Martins Sallun e Auxílio à Pesquisa (Processos 03/09681-9 e 03/13229-4). Ao CNPq, pela concessão de Bolsa de Doutorado a Janaina Santos (Processo 140316/2004-3), de Produtividade a Kenitiro Suguio (Processo 304718/2003-3) e à Sonia Hatsue Tatumi (Processo 303974/2005-2).

\section{Referências}

Aitken M. J. 1985. Thermoluminescence dating. London, Academic, $359 \mathrm{p}$.

Aitken M.J. 1998. An introduction to optical dating. London, Oxford University Press, $230 \mathrm{p}$.

Arruda E.M. 2004. Caracterização dos ambientes deposicionais na Bacia do Ribeirão Entupido, Complexo Alcalino de Passa Quatro, Estado de São Paulo. Dissertação de Mestrado, Instituto de Geociências e Ciências Exatas, Universidade Estadual Paulista, 174 p.

Barcena J.L., Urbina M., Rowlands A.P., Beneitez P., Millan A., Calderon T. 1999. Basic thermoluminescence properties of micas: muscovite, sericite and phlogopite. Radiat. Prot. Dosim., 84:289-292.

Bailiff I.K. 1976. Use of phototransfer for the anomalous fading of thermoluminescence. Nature, 264:531-533.

Barreto A.M.F. 1996. Interpretação paleoambiental do sistema de dunas fixadas do médio Rio São Francisco, Bahia. Tese de Doutoramento, Instituto de Geociências, Universidade de São Paulo, 174 p.

Barreto A.M.F., Angulo R.J., Tatumi S.H., Watanabe S., Ayta W.E.F. 1999. Datações por termoluminescência (TL) de sedimentos da planície costeira de Paranaguá, Estado do Paraná. In: ABEQUA, Cong. Bras. Ass. Bras. Est. Quat., 7, Boletim de Resumos, CD-ROM.

Barreto A.M.F., Suguio K., De Oliveira P.E., Tatumi S.H. 2002a. Campo de dunas inativas do médio Rio São Francisco - Marcante registro de ambiente desértico do Quaternário Brasileiro. In: C. Schobbenhaus (ed.) Sítios geológicos e paleontológicos do Brasil. SIGEP-DNPMCPRM, p. 223-231.

Barreto A.M.F., Bezerra F.H.R., Suguio K., Tatumi S.H., Yee M., Paiva R.P., Munita C.S. 2002b. Late Pleistocene marine terrace deposits in northeastern Brazil: sea-level change and tectonic implications. Palaeogeogr. Palaeoclim. Palaeoecol., 179:57-69.

Barreto A.M F., Suguio K., Bezerra F.H.R., Tatumi S.H., Yee M., Giannini P.C. 2004. Geologia e geomorfologia do
Quaternário Costeiro do Estado do Rio Grande do Norte. Geol. USP Sér. Cient., 4:1-12.

Bateman M.D., Frederick C.D., Jaiswal M.K., Singhvi A.K. 2003. Investigations into the potential effects of pedoturbation on luminescence dating. Quat. Sci. Rev., 22:11691176.

Bateman M.D., Boulter C.H., Carr A.S., Frederick C.D., Peter D., Wilder M. 2007. Preserving the palaeoenvironmental record in drylands: bioturbation and its significance for luminescence-derived chronologies. Sed. Geol., 195(1/2):5-19.

Bentz D. 2004. Os cordões litorâneos da planície de UnaJuréia, municípios de Peruíbe e Iguape, SP. Dissertação de Mestrado, Instituto de Geociências, Universidade de São Paulo, 108 p.

Berger G.W. \& Huntley D.J. 1983. Dating volcanic ash by thermoluminescence. PACT, 9:581-592.

Berger G.W. \& Busacca A.J. 1995. Thermoluminescence dating of late Pleistocene loess and tephra from eastern Washington and southern Oregon and implications for the eruptive history of Mount St. Helens. Jour. Geoph. Res., 100(B11):22361-22374.

Bizzi L.A., Schobbenhaus C., Gonçalves J.H., Baars F.J., Delgado I. M., Abram M.B., Leão Neto R., Matos G.M.M., Santos J.O.S. 2001. Geologia, tectônica e recursos minerais do Brasil: Sistema de Informações Geográficas - SIG e mapas na escala 1:2.500.000. Brasília, CPRM, 4 CD-ROM.

Botter-Jensen L., McKeever S.W.S., Wintle A. 2003. Optically Stimulated Luminescence Dosimetry. Amsterdam, Elsevier Books, 355 p.

Carneiro Filho A., Schwartz D., Tatumi S.H., Rosique T. 2002. Amazonian paleodunes provide evidence for drier climate phases during the Late Pleistocene-Holocene. Quat. Res., 58:205-209.

Chen R. \& McKeever S.W.S. 1997. Theory of Thermoluminescence and Related Phenomena. World Scientific, 
Singapore, $454 \mathrm{p}$.

Corrêa A.C.B., Barreto A.M.F., Bezerra, F.H.R., Neves B.B.B., Suguio K. 2005. Análise geomorfológica e sedimentológica do Graben de Cariatá, PB. In: ABEQUA, Cong. Bras. Ass. Bras. Est. Quat., 10, Boletim de Resumos, CD-ROM.

De Corte F., Vandenberghe D., Buylaert J.-P., Van den Haute P., Kucera J. 2006. Relative and $\mathrm{k}_{0}$-standardized INAA to assess the internal (Th, $\mathrm{U}$ ) radiation dose rate in the "quartz coarse-grain protocol" for OSL dating of sediments: Unexpected observations. Nuc. Instr. Meth. Phys. Res., 564(2):743-751.

Dillenburg S.R. 1994. A laguna de Tramandaí: Evolução geológica e aplicação do método geocronológico da termoluminescência na datação de depósitos sedimentares lagunares. Tese de Doutoramento, Universidade Federal do Rio Grande do Sul, 142 p.

Duller G.A.T. 2004. Luminescence dating of Quaternary sediments: recent advances. Jour. Quat. Sci., 19:183192.

Fett Júnior N. \& Oliveira M.A.T. de. 2005. Depósitos quaternários de planície de inundação no curso médio do Rio Pardo, Candelária, Rio Grande do Sul. In: ABEQUA, Cong. Bras. Ass. Bras. Est. Quat., 10, Boletim de Resumos, CD-ROM.

Gemmell A.M.D. 1994. Environmental controls on the TL age of modern zero-age proglacial outwash sediments. Quat. Geoch., 13:485-489.

Geyh M.A. \& Schleicher H. 1990. Absolute age determination. Berlin, Springer-Verlag, 503 p.

Giannini P.C.F., Guedes C.C.F., Assine M.L., Angulo R.J., Souza M.C., Pessenda L.C.R., Tatumi S.H. 2003. Variação transversal e longitudinal de propriedades sedimentológicas nos cordões litorâneos da ilha Comprida, litoral sul paulista. In: ABEQUA, Cong. Bras. Ass. Bras. Est. Quat., 9, Boletim de Resumos, CD-ROM.

Goble R.J., Mason J.A., Loope D. B., Swinehart J.B. 2004. Optical and radiocarbon ages of stacked paleosols and dune sands in the Nebraska Sand Hills, USA. Quat. Sci. Rev., 23:173-1182.

Grögler N., Houtermans E.G., Stauffer H. 1958. Radiation damage as a research tool for geology and prehistory. In: RIENSN, Conv. Sul. Dotaz. Met. Nucl., Proceedings, p.5-15.

Hole F.D. 1981. Effects of animals on soil. Geoderma, 25:75112.

Huntley D.J. 1985. On the zeroing of the thermoluminescence of sediments, Phys. Chem. Min., 12:122-127.

Huntley D.J., Hutton J.T., Prescott J.R. 1993a. The stranded beach-dune sequence of South-East South Australia: a test of thermoluminescence dating, 0-800 ka. Quat. Sci. Rev.,12:1-20.

Huntley D.J., Hutton J.T., Prescott J.R. 1993b. Optical dating using inclusions within quartz grains. Geology, 21:10871090.

Huntley D.J. \& Lamothe M. 2001. Ubiquity of anomalous fading in K-feldspars and the measurement and correction for it in optical dating. Can. Jour. Ear. Sci., 38:10931106.

Ikeya M., Filho B.O., Mascarenhas S. 1984. ESR dating of cave deposits from Akiyoshi-dô cave in Japan and Diabo cavern in Brazil. J. Speleol. Soc. Jap, 9:58-67.

Jeong G. Y., Cheong C., Choi J. 2007. The effect of weathering on optically stimulated luminescence dating. Quat. Geoch., 2(1-4):272-277.

Lamothe M \& Auclair M. 1999. A solution to anomalous fading and age shortfalls in optical dating of feldspar minerals. Ear. Plan. Sci. Lett., 171:319-323.

Lamothe M \& Auclair M. 2000. The fadiga method: a new approach in luminescence dating using the analysis of single feldspar grains. Rad. Meas., 32:433-438.

Li S-H. \& Wintle A.G. 1992. Luminescence sensitivity change due to bleaching of sediments. Nucl. Tracks Radiat. Meas., 20(4):567-57.

Li S-H. 1994. Optical dating: insufficiently bleached sediments. Rad. Meas., 23:563-568.

Li S-H. \& Yin G-M. 2006. Luminescence properties of biotite relevant to dating and dosimetry. Jour. Lumin., 121:51-56.

Lian O.B. \& Roberts R.G. 2006. Dating the Quaternary: progress in luminescence dating of sediments. Quat. Sci. Rev., 25(19/20):2449-2468.

Maia L. P., Rodrigues A.C.B., Cascon H.M., Barreto A.M.F., Castro I. de B., Tatumi S. H., Costa A.A. 1999. Correlação estratigráfica em poços de subsuperfície e datação de dunas costeiras inativas na região do Cauípe e Pecém - Costa Oeste do Ceará. In: ABEQUA, Cong. Bras. Ass. Bras. Est. Quat., 7, Boletim de Resumos, CD-ROM.

Mejdahl V. 1979. Thermoluminescence dating: beta-dose attenuation in quartz grains. Archaeometry, 21:61-72.

Miallier D., Gibert F., Faïn J., Pilleyre Th., Sanzelle S. 2001. Fluid inclusions in quartz: interference with thermoluminescence and its application to dating. Quat. Sci. Rev., 20:901-905.

Murray A.S. \& Olley J.M. 2002. Precision and accuracy in the optically stimulated luminescence dating of sedimentary quartz: a status review. Geochronometria, 21:1-16.

Murray A.S. \& Wintle A.G. 2000. Luminescence dating of quartz using an improved single-aliquot regenerativedose protocol. Rad. Meas., 32:57-73.

Oliver R.L. 1990. Optical properties of waters in the MurrayDarling Basin, southeastern Australia. Austr. Jou. Mar. Fres. Res., 41:581-601.

Olley J.M., Murray A., Roberts R.G. 1996. The effects of disequilibria in the uranium and thorium decay chains on burial dose rates in fluvial sediments. Quat. Sci. Rev., 15:751-760.

Porat N., Levi T., Weinberger R. 2007. Possible resetting of quartz OSL signals during earthquakes - evidence from late Pleistocene injection dikes, Dead Sea Basin, Israel. Quat. Geoch., 126(2):303-308.

Poupeau G., Souza J. H., Soliani Jr. E.L., Loss E.L. 1984. Dating quartzose sands of the Coastal Province of Rio Grande do Sul, Brazil, by thermoluminescence. Pesquisas, 16:250-268.

Poupeau G., Soliani Jr. E.L., Rivera A., Loss E.L., Vasconcellos M.B.A. 1988. Datação por termoluminescência de alguns depósitos arenosos costeiros, do Último Ciclo Climático, no nordeste do Rio Grande do Sul. Pesquisas, 21:25-47. 
Prescott J.R. \& Robertson G. B. 1997. Sediment dating by luminescence: a review. Rad. Meas., 27(5/6):893-922.

Prescott J.R. \& Stephan L.G. 1982. The contribution of cosmic radiation to the environmental dose for thermoluminescent dating, latitude, altitude and depth dependences. PACT, 6:17-25.

Rhodes E.J. \& Pownall L. 1994. Zeroing of the OSL signal in quartz from young glaciofluvial sediments. Rad. Meas., 23:581-586.

Sallun A.E.M., Suguio K., Tatumi S. H., Yee M. 2006. Dosimetria da radiação cósmica por espectroscopia-gama e datação de depósitos cenozóicos da porção central da Bacia Hidrográfica do Rio Paraná (MS, PR e SP). In: SBG, Cong. Bras. Geol., 43, Anais, p.321.

Sallun A.E.M. \& Suguio K. 2006. Datação absoluta da Aloformação Paranavaí (SP, PR e MS) por luminescência. Rev. IG (no prelo).

Santos J.C. dos, Barreto A.M.F., Suguio K. 2005. Caracterização geológica dos depósitos coluviais do Parque Nacional da Serra da Capivara, Piauí. In: ABEQUA, Cong. Bras. Ass. Bras. Est. Quat., 10, Boletim de Resumos, CDROM.

Shelkoplyas V.N. \& Morozov G.V. 1965. Some results from in investigation of Quaternary deposits by the thermoluminescence method. In: INQUA, Inter. Quat. Assoc. Cong., 7, Proceedings, p. 83-90.

Srivastava P., Brook G.A., Marais E., Morthekai P., Singhvi A.K. 2006. Depositional environment and OSL chronology of the Homeb silt deposits, Kuiseb River, Namíbia. Quat. Res., 65:478-491.

Stevaux J.C. 1993. O Rio Paraná: geomorfogênese, sedimentação e evolução quaternária de seu curso superior (região de Porto Rico, PR). Tese de doutoramento, Instituto de Geociências, Universidade de São Paulo, 242 p.

Stevaux J.C. 2000. Climatic events during the Late Pleistocene and Holocene in the Upper Paraná River: correlation with NE Argentina and South-Central Brazil. Quat. Inter, 72:73-85.

Suguio K., Tatumi S.H., Kowata E.A., Munita C.S., Paiva R.P. 2003. Upper Pleistocene deposits of the Comprida Island (São Paulo State) dated by thermoluminescence method. An. Ac. Bras. Cienc., 75:91-96.

Szmuk P.E. \& Watanabe S. 1971. Dating of brazilian indian pottery. TLD Publ. IEA., 231, 7 p.

Takada M., Tani A., Shimada A. 2006. Preliminary study of the application of natural olivine in cenozoic dating. Rad. Meas., 41:982-986.

Tatumi S.H.1994. Estudo das propriedades termoluminescentes e dos centros paramagnéticos da aragonita. Tese de Doutoramento, Instituto de Física, Universidade de São Paulo, 116 p.

Tatumi S. H., Batista L.R.,Watanabe S., Matsuoka M. 1989. Thermoluninescence dating of calcite deposits in a brasilian cave. Nucl. Instr. Meth. in Phis. Res., A280:510513.
Tatumi S.H., Gozzi G., Kowata E.A., Kassab L.R.P., Brito S.L. M., Peixoto M. N. O., Moura R.S., J., Mello C.L., Carmo I.O. 2003a. Optical dating using feldspar from quaternary alluvial and colluvial sediments from SE Brazilian Plateau, Brazil. Jour. Lumin., 102(103-C):566570.

Tatumi S.H., Kowata E.A., Gozzi G., Kassab L.R.P., Suguio K., Barreto A.M.F., Bezerra F.H.R. 2003b Optical dating results of beachrock, eolic dunes and sediments applied to sea-level changes study. Jour. Lumin., 102:562-565.

Tatumi S. H., Gozzi G.,Yee M., Oliveira V.I., Sallun A.E.M., Suguio K. 2006. Luminescence dating of quaternary deposits in geology in Brazil. Rad. Protec. Dos., 119(1-4): 462-469.

Thomas D.S., Brook G., Shaw P., Bateman M.D., Haberyan K., Appleton C., Nash D., McLaren S., Davies F. 2003. Late Pleistocene wetting and drying in the NW Kalahari: an integrated study from the Tsodilo Hills, Botswana. Quat. Intern., 104:53-67.

Tsirliganis N.C., Polymeris G.S., Kitis G., Pagonis V. 2007. Dependence of the anomalous fading of the TL and blueOSL of fluorapatite on the occupancy of the tunnelling recombination sites, Jour. Lum., in press (doi:10.1016/ j.jlumin.2006.07.011).

Ugumori T. \& Ikeya M., 1980. Luminescence of $\mathrm{CaCO}_{3}$ under $\mathrm{N}_{2}$ laser excitation and application to archaeological dating. Jpn. J. Appl. Phys., 19:459-465.

Wagner G.A. 1998. Age determination of young rocks and artifacts: physical and chemical clocks in Quaternary geology and archaeology. Berlin and Heidelberg, Springer-Verlag, $466 \mathrm{p}$.

Wallinga J., Murray A.S., Duller G.A.T., Tornqvist T.E. 2001. Testing optically stimulated luminescence dating of sand-sized quartz and feldspar from fluvial deposits. Ear. Plan. Sci. Let., 193:617-630.

Watanabe S., Ortega N.R.S., Feria Ayta W.E., Coaquira J.A.H., Cortezão S.U., Arenas J.S.A. 1997. TL dating of sands from Ilha de Cananéia. Radiat. Meas., 27(2):373376.

Wintle A.G. 1997. Luminescence dating: laboratory procedures and protocols. Radiat. Meas., 27:769-817.

Wintle A.G. \& Huntley D.J. 1980. Thermoluminescence dating of ocean sediments. Can. Jour. Ear. Scie., 17:348360 .

Wintle A.G. \& Huntley D.J. 1982. Thermoluminescence dating of sediments. Quat. Sci. Rev., 1:31-53.

Wintle A.G. \& Murray A.S. 2006. A review of quartz optically stimulated luminescence characteristics and their relevance in single-aliquot regeneration dating protocols. Rad. Meas., 41:369-391.

Manuscrito ID 7800

Submetido em 25 de abril de 2007 Aceito em 09 de dezembro de 2007 Sistema eletrônico de submissão 